\title{
ESTIMATION OF CURRENT DENSITY USING HIGH-SPEED-CAMERA RECORDINGS IN A MODEL SPARK GAP DURING SURGE CURRENTS
}

\author{
T. H. KopP*, E. Peters, M. Kurrat \\ Institute for High Voltage Technology and Electrical Power Systems, Technische Universität Braunschweig, \\ Braunschweig, Germany \\ * t.kopp@tu-braunschweig.de
}

\begin{abstract}
For experimental investigations of short time plasma in spark gaps, as used in surge protective devices, high-speed camera recordings are used frequently. The analysis of these recordings provides further details regarding the plasma state and distribution. These deduced details are used to assist research and development processes.

To increase the benefit of high-speed camera recordings an empirical model is presented to improve the picture analysis. In this model the recorded radiation intensity is empirically related to the current density within a spark gap. Therefore a specially adapted model spark gap was developed and tested. In this model spark gap areas with homogenous current densities occur. These current densities are determined in the experimental setup through current measurements with separated electrodes. Additionally, the relative radiation intensity between the electrodes is identified using high-speed camera recordings. An empirical correlation between these two measurement values was found and is discussed. It confirmed that the determined correlation improve the mostly intuitive interpretation of high speed camera recordings in spark gaps.
\end{abstract}

Keywords: spark gap, plasma, surge protective device, high speed camera, current density, narrow gap.

\section{Introduction}

Surge protective devices, SPD class 1, are required within power grids to ensure the normal operation of the electrical installation [1]. With these devices surges are driven directly to the equipotential bonding bypassing the electrical installation. In order to investigate the conductive behavior of SPD's during operation, they are tested using current generators, which deliver standardized $8 / 20 \mu$ s pulse currents according to international standards [2].

Spark gaps are used as class 1 SPD's. Within spark gaps an electric arc forms the bypassing path during the protecting operation $[3,4]$. During this process the main aspects are as follows: Firstly, SPD's have to create a highly conductive path to divert the surge current to the equipotential bonding. Thus, the protection of the connected electric devices is guaranteed. Secondly, the conductivity of the plasma has to be reduced by several orders of magnitude after the surge, in order to prevent the subsequent fault current fed by the power network [4]. These requirements are opposing each other and pose a challenge to undertake.

The behavior of the spark gap depends on the plasma properties and it's propagation within the plasma chamber of the model spark gap. To improve the protective function this behavior has to be known for the development process [2].

Hence information regarding the plasma distribution is necessary to investigate the switching behavior of spark gaps. In present research of SPD's, high speed camera recordings can be used to visualize the distri- bution and support investigations in modern research and development projects [5-9].

In actual fundamental research projects information regarding radiation and temperature [10], pressure [11], and electrical conductivity [12] are given for the transient time domain in spark gaps. These data are not yet measured simultaneously and therefore cannot verify a complete plasma model. However, during the development processes of spark gaps, empiric models are often sufficient to estimate the plasma state and to identify the changes caused by development steps of the SPD design.

The method presented in this publication shall provide an additional tool for analysis and evaluation of high speed camera recordings in spark gaps. As a result the current density can be deduced quickly from the high-speed camera recording of a spark gap. It is the aim to support the development processes of spark gaps. Therefore, it is sufficient to provide a basic empirical relation between the recordings and the current density. However the presented method is applicable for spark gaps due to their behavior being comparable to a black or grey radiator. For line radiators the application is not recommended.

The method, section 2, is divided into four subsections: description of the measurement setup and of the model spark gap, an introduction into the theoretic approach and finally the data acquisition.

To assess the applicability of the method, measurement results are given in section 3. Here, the possibilities of this method are presented while discussing the 
change of the current density in the plasma during a surge current.

In section 4 the functionality of this method will be discussed and an outlook for further investigations will be given.

\section{Method}

A measurement setup was built to investigate the current density in relation to high speed camera recordings of a model spark gap as shown in Fig. 1. The current source of this measurement circuit is an impulse generator generating an unipolar surge current up to $20 \mathrm{kA}$, with a rise time of $8 \mu \mathrm{s}$ and a time to half-value of $20 \mu \mathrm{s}$, according to the international test standard [2].

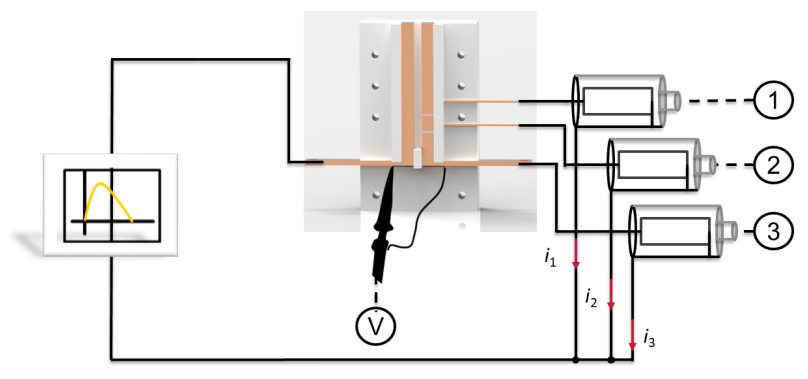

Figure 1. Measurement setup.

The measurement system provides a 14-bit amplitude resolution and a sampling rate of $100 \mathrm{MS} / \mathrm{s}$ [9, 11]. In this setup the surge current is separated inside the spark gap through different electrodes, therefore the partial currents $i_{1}$ to $i_{3}$ are measured separately with three coaxial shunts of the same type. The shunts have a resistance of $1 \mathrm{~m} \Omega$ and show low inductive behavior. The voltage at the connector of the spark gap $u_{\text {arc }}$ is measured with a high voltage probe (Type PMK $3.6 \mathrm{kV})$.

A model spark gap is used as device under test in this measurement setup [5], Fig. 1. The spark gap consists of one electrode (anode) and three separated electrodes $\mathrm{C}$ (cathode), they are connected with the measurement setup via copper bus bars, Fig. 2. The distance of the anode and cathode is $5 \mathrm{~mm}$ and the separated cathodes are isolated with a $1 \mathrm{~mm}$ thick polymer layer.

The plasma chamber $\mathrm{P}$ is surrounded with evaporating polymer and has a height of $80 \mathrm{~mm}$, a width of $5 \mathrm{~mm}$ and a depth of $1 \mathrm{~mm}$. The chosen polymer is poly-oxy-methylene (POM). To obtain optical access to the plasma chamber in the front of the spark gap a cover of poly-methyl-methacrylat (PMMA) is used.

The plasma was ignited using a spark discharge device (SDD) which is connected in series with a varistor to ensure a response with occurring high voltages.

The plasma radiance in the plasma chamber was recorded with a high speed camera of the type Motion Pro X4 from the manufacturer Redlake with a CMOSsensor. The sensor has a resolution of 8 bit with an pixelarea of $16 \mu \mathrm{m}$ x $16 \mu \mathrm{m}$. During all measurements

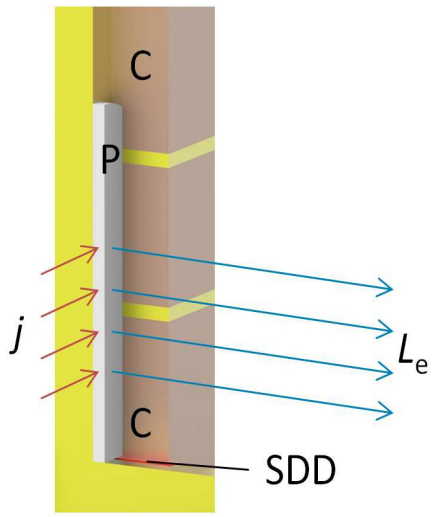

Figure 2. Model spark gap with separated cathode.

an integration time of $1 \mu \mathrm{s}$ was applied. The frame rate for the recording was set to $94.500 \mathrm{fps}$. Different settings for aperture and neutral density filters were used for different recorded surge currents amplitudes, as considered in the analysis.

The plasma distribution between the electrodes depends on the surge current amplitude and the geometrie model spark gap. Within the following introduction into the theoretical approach firstly the relation between electrical conductivity and radiance has to be determined. This dependency could be deduced applying suitable material properties [13]. In order to do so, the temperature must be derived from its spectral radiance. Recent investigations with spark gaps have shown that, due to the high plasma pressure, the plasma has a low optical depth and therefore the radiance assume to be same as for a grey body radiator [10, 14], Fig. 3. In the respective spectra it should be noted that PMMA has a cut off wavelength by approximately $375 \mathrm{~nm}$.

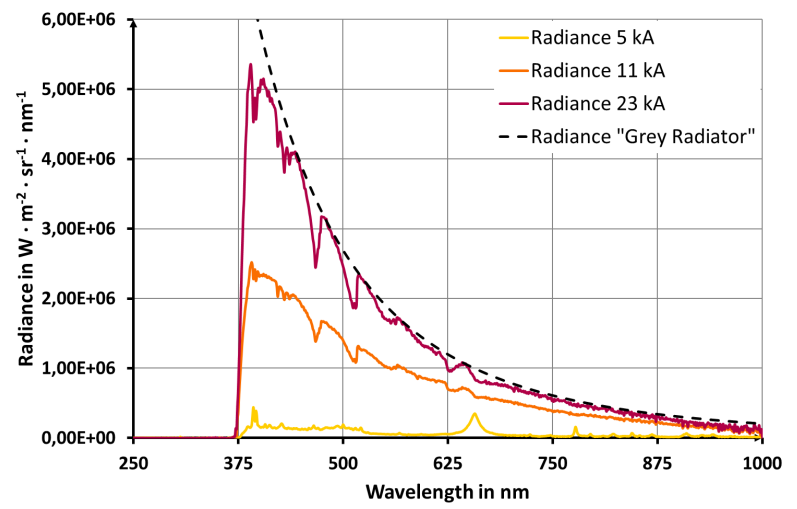

Figure 3. Spectral Radiance acc. to [14].

Thus, the temperature can be determined by comparing the measured radiance to the theoretical spectral radiance of a grey radiator with a suitable emission coefficient, which is between 0.8 and 1 [14]. Furthermore, the PMMA cover, the quantum efficiency of the sensor and the adjusted filters have to be considered. While using a high speed camera as radiance sensor, the recieved spectral radiance is converted to 
the recieved radiance. This is done by integrating the spectral radiance over the wavelength range of the sensitivity of the camera. With this radiance the temperature can be estimated and subsequently the electrical conductivity. However, a necessary requirement to compute the conductivity is the information of plasma pressure for the spark gap, as given in [11]. For spark gaps it is known that the pressure rises linearly with increasing current density [11]. Combining radiance and pressure the relation to the electrical conductivity can be deduced in more detail using the material properties [13], Fig. 4.

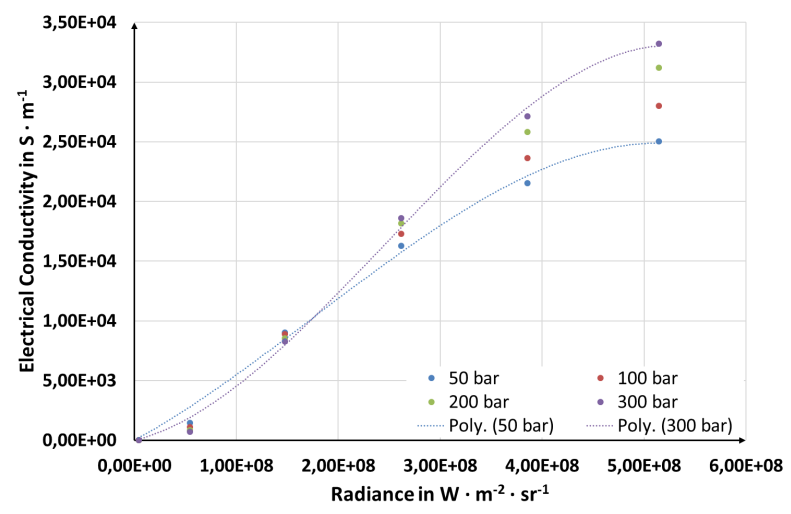

Figure 4. : Relation between electrical conductivity and radiance.

With the deduced conductivity and the geometry of the spark gap, the current density can be determined. However a measured arc column voltage $u_{\text {arc }}$ (under consideration of the induced voltage [15]) and the voltage of the electrode sheath $u_{\mathrm{es}}$, is also necessary for the implementation in a simplified approach (1):

$$
j=\sigma(p, T) \cdot \frac{u_{\mathrm{arc}}-u_{\mathrm{es}}}{l_{\mathrm{arc}}}
$$

In this brief theoretical approach the dependence between current density (1) and radiation Fig. 3 (hence temperature) was shown. Due to the presented complex correlation between this quantities only an empirical approach will be used in the next chapter "Results". There, with the high speed camera recordings the correlation from the radiance to the current density is derived for different surge current amplitudes (5, 10 and $20 \mathrm{kA}$ ). For each current, three measurement series are done. Within each series ten surge currents was applied to an unused spark gap.

To develop an empirical model the current in the lower section of the spark gap (shunt 3, Fig. 1) was correlated with the mean value of the radiance of this area recorded by the high speed camera during its integration periode. This area has a width of $5 \mathrm{~mm}$ and a height of $10 \mathrm{~mm}$. It is optically recorded from a distance of approx. $1.5 \mathrm{~m}$ on $12 \times 24$ pixel. The lower section has the advantage that information regarding the pressure was recorded in [11]. Furthermore, the current density can be assumed in this area as homogeneously distributed [5].
The mean radiance is considered relative to the maximum recorded brightness (255 bit number) with a transmission factor of $12.5 \cdot 10^{-6} \%$ (aperture 32 and neutral density filters, ND 3.0 and ND 0.6).

\section{Results}

With the camera recordings and the data of the measured current with the shunt, the correlation between current density and relative radiance is determined. The relative radiance is the quotient of the mean radiance in the lower section to the maximum recordable brightness. The mean radiation was calculated by averaging the pixel values in the area of $12 \times 24$ pixel. Regarding the current densities and the radiance of every recording an empirical correlation can be found, Fig. 5.

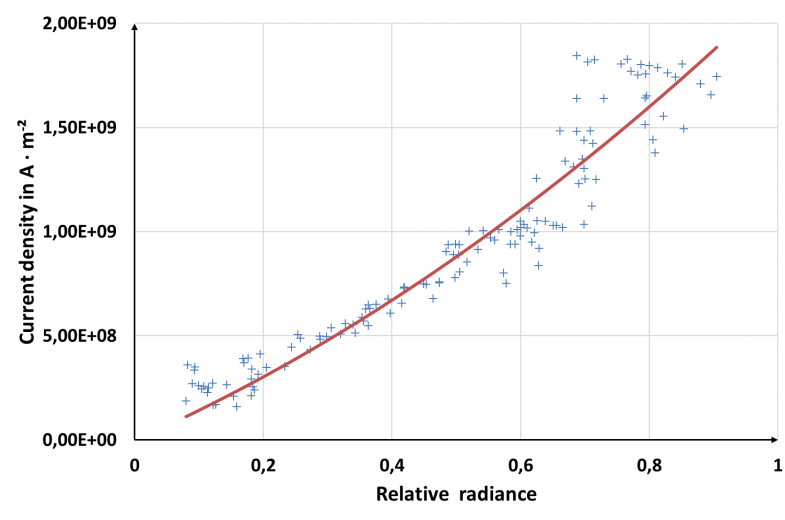

Figure 5. Correlation Current density and relative radiance.

Within this data plot 5, 10, and $20 \mathrm{kA}$ measurements are considered. It is shown that with higher radiance the current density increases as well. This correlation can be described approximately using the square formula (2) with the current density $j$ and the relative radiance $L_{\mathrm{r}}$ :

$$
j=8 \cdot 10^{8} \cdot L_{\mathrm{r}}^{2}+1.36 \cdot 10^{9} \cdot L_{\mathrm{r}}
$$

Formula (2) has a determination-coefficient of 0.913 to the recorded data. The relation between current density and radiance can be explained according to the shown theoretical approach (chapter 2). Exemplary this relation has been verified with measurement data of a reference spark gap which is discussed in [14]. For this reference geometry the relation of the radiance recorded with optical emission spectroscopy (OES) as published in [10] to the measured current density is evaluated, Fig. 6. To increase the significance of the presented model, the reference spark gap was used. This improving is obtained due to same material (POM) and a slightly different geometry of the reference spark gap.

Within these OES-data the optical transmission of the real optical path is considered. As a result the reference spark gap shows qualitatively similar results 


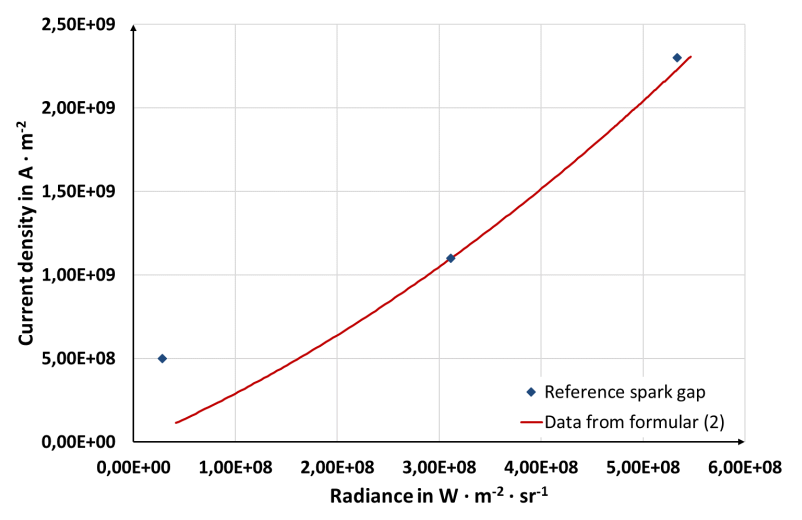

Figure 6. Correlation Current density and radiance for reference spark gap.

as the measurements with the high speed camera system. Therefore, the method to link the irradiance on the camera chip with the current density is considered applicable. The deviation of the curve at low current density is due to the spectral behavior observed for the $5 \mathrm{kA}$ measurement (Fig. 3), whose spectrum is more like a line than a Planck radiator. Therefore the application of the method appears to have a bigger uncertainty for surge currents of lower amplitude.

Within the recorded data, at an occurring relative radiance over 0.6, the scattering is increasing (Fig. 5). In theory [14] the temperature determination based on the grey body model also shows differences according to a model based on transport properties. The method could be more accurate if the transport properties are taken into account. However, the aim to provide an easily and quickly applicable empirical model with a comprehensible theoretical behavior is achieved. The grey body radiation is sufficient due to a function uncertainty of $30 \%$. For more accurate investigations the transport properties have to be considered.

\section{Conclusions and Outlook}

In this publication a method for current density estimation in spark gaps is presented. It is shown that the radiance during measurements with different surge currents can be empirically related to the current density. Furthermore, the empirically described relation shows also comparable patterns with the spectral data of the reference spark gap.

The approach was tested in this contribution with two different designs of model spark gaps. Yet, the empirical function still has to be verified with other types of spark gaps. This method can preferably be applied when the radiative characteristics of the plasma are like those of a grey or black body radiator. Due to the behavior of the plasma as a grey body radiator this method should be applicable also for spark gaps with other plasma geometrial arrangements. However, in further contributions the boundaries of the method has to be investigated to give a statement of the applicability under different circumstances.

\section{Acknowledgements}

The authors gratefully thank the company Phoenix Contact $\mathrm{GmbH}$ and Co. KG for the financial and technical support. Additionally we thank F. Fitzel M. Sc. for the help during the execution of the experiments and the discussions during the writing of his bachelor thesis. In particular we specially thank Dr.-Ing. T. Runge for his efforts and valuable discussion.

\section{References}

[1] Electrical installations of buildungs - Part 5-53: Selection and erection of electrical equipment Isolation, switching and control. Geneva, Switzerland, 2002. IEC 60364-5-53.

[2] Surge protective devices connected to low-voltage power systems - Requirements and test methods. Geneva, Switzerland, 2011. IEC 61643-11.

[3] L. Hüttner, L. Jurcacko, F. Valent, A. Ehrhardt, S. Schreiterand, and M. Rock. Fundamental analysis of encapsulation of a low-voltage spark gap with deion chamber. In XVIX ${ }^{\text {th }}$ Symposium of Physics of Switching Arc, Nove Mesto na Morave, Czech Republic, 2011.

[4] G. Finis, R. Durth, C. Depping, and M. Wetter. Laboratory for the qualification-testing of spds combining highest power performance parameters with unique fine adjustment possibilities. In International Conference on Lightning Protection (ICLP), Shanghai, China, 2014. doi:10.1109/ICLP. 2014.6973145.

[5] B. Schottel. Plasmavorgänge im engen Spalt beim Ableiten hoher Stoßströme bis $25 \mathrm{kA}$. PhD thesis, Technische Universität Braunschweig, 2014.

[6] A. Ehrhardt and S. Beier. New deion chamber for encapsulated switchgear. In $27^{\text {th }}$ International Conference on Electric Contacts, Dresden, Germany, 2014.

[7] S. Ait-Amar, G. Serrie, J. B. Docourneau, and M. Abplanalp. Arc extinguishing method of spd type 1 . In $29^{\text {th }}$ International Conference on Lightning Protection, Uppsala, Schweden, 2008.

[8] B. Schottel, T. H. Kopp, T. Runge, and M. Kurrat. Experimental investigation of short-time plasma propagation recorded by high speed camera. In International Conference on Gas Discharges and their Applications, Orleans, France, 2014.

[9] T. Kopp, T. Runge, and M. Kurrat. Analysis of arc behavior in a model spark gap after surge currents. IEEE Transactions on Components, Packaging and Manufacturing Technology, 8(6):958-965, 2018. doi:10.1109/TCPMT . 2018.2811940.

[10] T. Runge, S. Franke, S. Gortschakow, and M. Kurrat. Optical investigations on plasma temperature estimation in a model spark gap for surge currents. Plasma Physics and Technology, 4(2):108-111, 2017. doi:10.14311/ppt.2017.2.108.

[11] T. Runge, T. Kopp, and M. Kurrat. Novel approach for maximum plasma pressure estimation at surge current based on experimental investigations. IEEE Transactions on Plasma Science, 46(8):2935-2941, 2018. doi:10.1109/TPS.2018.2850364. 
[12] T. Runge, T. Kopp, and M. Kurrat. Experimental investigations on electrical plasma conductivity in a model spark gap for surge currents. Plasma Physics and Technology, 4(1):24-27, 2017.

doi:10.14311/ppt.2017.1.24.

[13] A. D'Angola, G. Colonna, C. Gorse, and M. Capitelli. Thermodynamic and transport properties in equilibrium air plasmas in a wide pressure and temperature range. The European Physical Journal, 46(1):129-150, 2008. doi:10.1140/epjd/e2007-00305-4.

[14] T. Runge. Plasmaeigenschaten in Funkenstrecken unter Stoßstrombelastung. $\mathrm{PhD}$ thesis, Technische Universität Braunschweig, 2018.

[15] O. Kerfin, T. H. Kopp, and M. Kurrat. Parasitic magnetic coupling in voltage measurement setups for impulse current tests. In International Symposium on Electromagnetic Compatibility EMC Europe, 2018. doi:10.1109/EMCEurope.2018.8485117. 